\title{
Elevated serum FGF21 predicts the major adverse cardiovascular events in STEMI patients after emergency percutaneous coronary intervention
}

\author{
Lingyun Gu ${ }^{1}$, Wenlong Jiang ${ }^{1}$, Huidong Qian ${ }^{1}$, Ruolong Zheng ${ }^{1}$, Weizhang Li ${ }^{\text {Corresp. } 1}$ \\ ${ }^{1}$ Department of Cardiology, Jiangyin hospital affiliated to Southeast University, Jiangyin, Jiangsu, China \\ Corresponding Author: Weizhang Li \\ Email address: liwz@jyrmyy.com
}

Background. Although there have been several studies related to serum fibroblast growth factor 21 (FGF21) levels and acute myocardial infarction, the value of serum FGF21 levels in ST-segment elevation myocardial infarction (STEMI) patients after emergency percutaneous coronary intervention (PCI) has not been previously investigated. Methods. A total of 348 STEMI patients who underwent emergency PCI were enrolled from January 2016 to December 2018. The primary endpoint was the occurrence of major adverse cardiovascular events (MACEs), with a median follow-up of 24 months. Eighty patients with stable angina (SA) who underwent selective PCl served as the control group. Serum FGF21 levels were measured by ELISA. Results. Serum FGF21 levels were significantly higher in the STEMI group than in the SA group $(225.03 \pm 37.98$ vs. $135.51 \pm 34.48, \mathrm{P}<0.001)$. Multiple linear regression analysis revealed that serum FGF21 levels were correlated with NT-proBNP $(P<0.001)$. According to receiver operating characteristic $(R O C)$ analysis, the areas under the ROC curve (AUCs) of FGF21 and NT-proBNP were 0.812 and 0.865 , respectively. The Kaplan-Meier curves showed that STEMI patients with lower FGF21 levels had an increased MACE-free survival rate. Cox analysis revealed that high FGF21 levels (HR: $2.011,95 \% \mathrm{Cl}: 1.160-3.489$ ) proved to be a powerful tool in predicting the risk of MACEs among STEMI patients after emergency PCl. Conclusion. Elevated FGF21 levels on admission have been shown to be a powerful predictor of MACEs for STEMI patients after emergency $\mathrm{PCl}$. 
1 Elevated serum FGF21 predicts the major adverse cardiovascular events in

2 STEMI patients after emergency percutaneous coronary intervention

4 Lingyun Gu, Wenlong Jiang, Huidong Qian, Ruolong Zheng, Weizhang Li

Department of Cardiology, Jiangyin hospital affiliated to Southeast University, Jiangyin, Jiangsu, China

9 Corresponding Author: Weizhang Li

10 Email address: liwz@jyrmyy.com

\section{Abstract}

13 Background. Although there have been several studies related to serum fibroblast growth factor 21 (FGF21) levels and acute myocardial infarction, the value of serum FGF21 levels in STsegment elevation myocardial infarction (STEMI) patients after emergency percutaneous coronary intervention (PCI) has not been previously investigated.

Methods. A total of 348 STEMI patients who underwent emergency PCI were enrolled from January 2016 to December 2018. The primary endpoint was the occurrence of major adverse cardiovascular events (MACEs), with a median follow-up of 24 months. Eighty patients with stable angina (SA) who underwent selective PCI served as the control group. Serum FGF21 levels were measured by ELISA.

Results. Serum FGF21 levels were significantly higher in the STEMI group than in the SA group (225.03 \pm 37.98 vs. $135.51 \pm 34.48, \mathrm{P}<0.001)$. Multiple linear regression analysis revealed that serum FGF21 levels were correlated with NT-proBNP $(\mathrm{P}<0.001)$. According to receiver operating characteristic (ROC) analysis, the areas under the ROC curve (AUCs) of FGF21 and NT-proBNP were 0.812 and 0.865 , respectively. The Kaplan-Meier curves showed that STEMI patients with lower FGF21 levels had an increased MACE-free survival rate. Cox analysis revealed that high FGF21 levels (HR: 2.011, 95\% CI: 1.160-3.489) proved to be a powerful tool in predicting the risk of MACEs among STEMI patients after emergency PCI. MACEs for STEMI patients after emergency PCI. adverse cardiovascular events 


\section{Introduction}

36 Acute myocardial infarction (AMI) is one of the most common cardiovascular emergencies, with

a high mortality and disability rate. An increasing number of studies have found that the

38

39 inflammatory response following AMI plays a significant role in determining the infarct area and subsequent adverse left ventricular (LV) remodeling (Ong S B, et al. 2018). As the understanding of inflammatory mechanisms following AMI has increased, many liver-derived biomarkers with excellent prognostic value, such as C-reactive protein and interleukin-6, have been discovered (Groot H E, et al. 2015; Ziakas A, et al. 2006).

Another liver-secreted cytokine with anti-inflammatory effects, fibroblast growth factor 21 (FGF21), has gained increasing interest in recent cardiovascular research. Previous studies have found that FGF21, in addition to its anti-inflammatory effects, has an antioxidative stress effect and regulates glycolipid metabolism (Fisher F M, et al. 2016; Luo Y, et al. 2017). Previous studies have found that inflammation, oxidative stress, and disturbances in glycolipid metabolism play an important role in the pathogenesis of AMI (Neri M, et al. 2017; Tao L, et al. 2015; Zhang Q, et al. 2018). Therefore, we speculate that FGF21 is correlated with the prognosis of AMI.

Previous studies have found that serum FGF21 levels were higher in patients with AMI than in patients with stable angina (SA), and correlated with the occurrence of major adverse cardiovascular events (MACEs) (Chen H, et al. 2018; Sunaga H, et al. 2019; Zhang W, et al. 2015). However, these studies did not distinguish between STEMI patients and NSTEMI patients. Therefore, the relationship between serum FGF21 levels and the prognosis of STEMI patients, who account for $45.8 \%$ of AMI patients (Kuch B, et al. 2007), remains unclear. The goal of this study was to evaluate whether serum FGF21 levels on admission could predict MACEs in STEMI patients after emergency percutaneous coronary intervention (PCI).

\section{Methods}

\section{Study population}

A total of 348 patients with STEMI admitted to Jiangyin People's Hospital were enrolled from January 2016 to December 2018. They all underwent emergency coronary angiography and PCI within 12 hours after the onset of ischemic symptoms. The STEMI patients received medication according to the American College of Cardiology Foundation/American Heart Association (ACCF/AHA) guidelines (O'Gara P T, et al. 2013). To exclude the influence of PCI on the experimental results, 80 patients with SA who underwent selective coronary angiography and PCI during the same period were selected as the control group. 
69

70

71

72

73

The research was approved by the Ethics Committee of Jiangyin People's Hospital (approval number: 2015ER035), and written informed consent was obtained from all enrolled patients before participation. STEMI is a clinical syndrome defined by characteristic symptoms of myocardial ischemia in association with persistent electrocardiographic (ECG) ST elevation and the subsequent release of biomarkers of myocardial necrosis (O'Gara P T, et al. 2013). Patients will be excluded if they present with the following diseases: cardiac shock, primary valvular heart disease, congenital heart disease, tachycardia-induced cardiomyopathy, pericardial disease, chronic liver insufficiency, acute renal failure, rheumatic disease, pulmonary embolism, neoplasm, inflammatory or infectious disorders, and excess alcohol consumption.

\section{Clinical and Laboratory Assessments}

The baseline characteristics of the participants and history of hypertension, diabetes mellitus and atrial fibrillation were recorded by experienced physicians. All patients underwent physical examination on admission, and elbow venous blood was drawn with a separation gel vacuum tube on an empty stomach early the next morning. A portion of the blood samples were sent to the central laboratory for troponin I testing by a Beckman DXI800 and for triglyceride, total cholesterol, low-density lipoprotein cholesterol (LDL-C), high-density lipoprotein cholesterol (HDL-C), uric acid, N-terminal proBNP (NT-proBNP) and creatinine assessment by Roche e602 and c701 modules, respectively. The other part of each blood sample was centrifuged to obtain serum and stored at $-80^{\circ} \mathrm{C}$ in a refrigerator, and serum FGF21 levels were measured every 3 months, as in our previous study (Gu L, et al. 2021).

\section{Echocardiography}

All patients underwent echocardiography on 7-10 days after primary PCI (P-PCI).

Echocardiogram was performed by experienced operators with an ultrasound machine (Philips iE 33 xMatrix).

The mitral regurgitation was examined and evaluated for severity using the flow convergence (PISA) method according to the guidelines of the American Society of echocardiography (Zoghbi W A, et al. 2003). Pulmonary artery pressure was assessed by the tricuspid regurgitation method. The left atrial diameter (LAD), interventricular septum thickness (IVST), left ventricular posterior wall thickness (LVPWT), left ventricular end-diastolic diameter (LVEDD), left ventricular end-systolic diameter (LVESD) and left ventricular ejection fraction (LVEF) were measured in the parasternal long-axis view in the M-mode images. left ventricular end-systolic volume (LVESV) and left ventricular end-diastolic volume (LVEDV) were measured by the Simpson method. Left ventricular mass (LVM) was calculated using the 
102 formula $0.8 \times 1.04 \times\left[\left(\right.\right.$ IVST $^{2} \mathrm{LVPWT}+\mathrm{LVEDD}^{3}{ }^{3}-\left(\mathrm{LVEDD}^{3}{ }^{3}\right]+0.6($ Lang R M, et al.

103 2015).

\section{Follow-up}

105 Follow-up, which was initiated at enrollment and ended in April 2020, was conducted for

106 STEMI patients only. The median follow-up time was 24 months (interquartile range, 16-36.25

107 months). The primary endpoint of the study was the first MACE, defined as all-cause mortality

108 or readmission for angina, heart failure, or recurrent AMI. The follow-up was conducted through

109 periodic outpatient interviews and scripted telephone interviews with the patients or their

110 families every month. Due to the high mobility of migrant workers and changes in phone

111 numbers, 30 of the STEMI patients were lost to follow-up. Among all STEMI patients, 106

112 recorded MACEs, including 6 deaths and 100 readmissions (Fig. 1).

\section{Statistical analysis}

114 Statistical analysis was conducted using the SPSS 22.0 statistical package. The quantitative

115 variables are expressed as the means \pm standard deviations and compared using Student's t test.

116 Categorical variables were expressed as absolute numbers (percentages) and compared using the

117 chi-square test and Mann-Whitney-Wilcoxon test. Multiple linear regression analysis was

118 performed on the STEMI groups to evaluate the association between serum FGF21 concentration

119 and other clinical covariates. Receiver operating characteristic (ROC) analysis was used to

120 determine the accuracy of the best cutoff value of variables for predicting the occurrence of

121 MACEs. MACE-free survival analysis was performed with Kaplan-Meier survival curves and

122 compared using the log-rank test. Univariate and multivariate Cox proportional hazards models

123 were used to analyze the relationships between FGF21 levels and MACEs by calculating hazard

124 ratios (HRs) and 95\% CIs. Multivariate Cox analysis was performed on all variables that reached

125 statistical significance in univariate Cox analysis. A p value $<0.05$ was considered statistically

126 significant (two-tailed).

127

\section{Results}

129 The demographic and baseline clinical characteristics of the subjects are summarized in Table 1.

130 No differences were observed in sex, age, incidence of atrial fibrillation, levels of triglycerides,

131 total cholesterol, uric acid, creatinine, pulmonary pressure, mitral regurgitation, IVST, LVPWT

132 and LVEDV between the STEMI and SA groups. Compared with the SA group, the STEMI

133 group had a higher incidence of hypertension and diabetes. LDL-C and NT-proBNP levels were

134 markedly higher in the STEMI group than in the SA group, while the HDL-C levels were lower

135 in the STEMI group than in the SA group (all $\mathrm{P}<0.05$ ). The LAD, LVEDD, LVESD, LVESV, 
136 LVM and LVEF were also markedly different between the groups. There were significant

137 differences between the two groups in the culprit artery and lesion site $(\mathrm{P}<0.05)$. The levels of

138 serum FGF21 were significantly higher in the STEMI group than in the SA group (225.03 \pm 37.98

139 vs. $135.51 \pm 34.48, \mathrm{P}<0.001$ ) (Table 1). Multiple linear regression analysis revealed that serum

140 FGF21 levels were correlated with NT-proBNP levels $(\mathrm{P}<0.001)$.

141 During the median follow-up of 24 months, 30 patients (8.62\%) were lost to follow-up.106

142 patients (30.46\%) experienced MACEs in the study: 6 patients died, and 100 patients were

143 rehospitalized due to angina, heart failure or recurrent AMI. According to the ROC analysis,

144 both FGF21 and NT-proBNP levels were significant indicators of MACEs in STEMI patients

145 (Fig. 2). The area under the ROC curve (AUC) of NT-proBNP was 0.865 (95\% CI: 0.822-0.909),

146 which was greater than that of FGF21 (AUC, 0.812; 95\% CI: 0.765-0.860). The difference

147 between the two AUCs was statistically significant $(\mathrm{Z}=2.230, \mathrm{P}=0.026)$.

148 According to the optimal cutoff value determined by ROC analysis and Youden index,

149 FGF21 was divided into high FGF21 level group and low FGF21 level group for Kaplan-Meier

150 analysis. The optimal cutoff value of FGF21 was $229.77 \mathrm{pg} / \mathrm{ml}$, with a corresponding sensitivity

151 of $84.00 \%$ and a specificity of $65.10 \%$.

152 In the Kaplan-Meier analysis, a significant difference was observed between the high serum

153 FGF21 level group and the low serum FGF21 level group (Fig. 3). The Mace-free probability

154 among STEMI patients with high serum FGF21 levels was significantly lower than that of

155 STEMI patients with low serum FGF21 levels.

156 The results of univariate and multivariate Cox proportional hazards models are shown in

157 Table 2. Univariate Cox analysis showed that a high FGF21 level was a predictor for MACEs in

158 STEMI patients, in addition to age, creatinine, troponin I, NT-proBNP, pulmonary pressure,

159 mitral regurgitation, LAD, LVESD, LVESV and LVEF. In the multivariate Cox analysis, high

160 FGF21 levels (HR: 2.224, 95\% CI: 1.122-4.407) also proved to be a powerful tool in predicting

161 the risk of MACEs. We also found that troponin I (HR: 6.842, 95\% CI: 3.870-12.094), NT-

162 proBNP levels (HR:3.452, 95\% CI: 1.765-6.750), and mitral regurgitation (HR: 2.011, 95\% CI:

163 1.160-3.489) increased the risk of MACEs.

164

\section{Discussion}

166 In this study, we demonstrated that serum FGF21 levels were significantly elevated in STEMI

167 patients and correlated with NT-proBNP. At a median follow-up of 24 months, MACE-free

168 survival was reduced in STEMI patients with elevated FGF21 levels, and elevated FGF21 levels

169 were shown to be a strong predictor of MACEs in STEMI patients. 
170 Recent studies have found that the heart is not only a target organ for FGF21 but also one of

171

172

173

174

175

176

177

178

179

180

181

182

183

184

185

186

187

188

189

190

191

192

193

194

195

196

197

198

199

200

201

202

203 its sources (Planavila A, Redondo-Angulo I, Ribas F, et al. 2015). The expression of FGF21 increases due to stimulation by inflammation, oxidative stress, lipid toxicity and endoplasmic reticulum stress, which plays an important role in cardiovascular disease (Brahma M K, et al. 2014: Planavila A, Redondo-Angulo I, Ribas F, et al. 2015; Steven S, et al. 2019). Then, FGF21 is synthesized by cardiomyocytes and secreted in an autocrine manner (Planavila A, et al. 2013; Planavila A, Redondo-Angulo I, \& Villarroya F 2015). In target cells, FGF21 binds to FGF receptors and $\beta$ Klotho, which are widely expressed in cardiomyocytes (Domouzoglou E M, et al. 2015). Increased cardiac FGF21 expression protects cardiomyocytes from inflammation, oxidative stress and dysfunction after myocardial I/R injury (Hu S, et al. 2018; Zhang J, et al. 2016).

Accumulating evidence indicates that elevated FGF21 concentrations are associated with a higher incidence of diabetes, chronic kidney disease, hypertension, and cardiovascular diseases, including coronary artery disease, heart failure, atrial fibrillation and AMI (Gu L, et al. 2020; Shen Y, et al. 2017; Shen Y, et al. 2018; Wang R, et al. 2015; Wu C H, et al. 2018; Zhang W, et al. 2015). Moreover, FGF21 significantly predicts the incidence of coronary artery disease and all-cause and cardiovascular mortality (Lakhani I, et al. 2018). This is consistent with the results of our study.

However, the increase in FGF21 levels, which has a cardioprotective effect, is negatively correlated with the prognosis of cardiovascular disease. Previous research reveals that the elevation of FGF21 levels in pathological conditions may be part of a compensatory response to the underlying metabolic stress or may be caused by FGF21 resistance as a result of impaired FGF21 signaling (Lewis J E, et al. 2019; Woo Y C, et al. 2013). Therefore, more FGF21 is secreted to compensate for its reduced metabolic regulation caused by FGF21 resistance. Moreover, additional supplementation with FGF21 has been shown to have a therapeutic effect in in vitro trials (Zhu W, et al. 2014). Furthermore, some companies, such as Eli Lilly and Pfizer, have now developed FGF21 analogs and demonstrated their therapeutic potential in clinical trials for the treatment of metabolic diseases (BonDurant L D, et al. 2018).

Inconsistent with previous studies, the Multi-Ethnic Study of Atherosclerosis (MESA) recently found that FGF21 levels were not associated with incident cardiovascular disease after multiple adjustments (Ong K L, et al. 2019); however, this study enrolled only patients without cardiovascular disease. In contrast to MESA, the patients enrolled in our study were STEMI patients, and we found that FGF21 was associated with the occurrence of MACEs. In MESA, 481 patients underwent PCI because of cardiac ischemia, mainly acute coronary syndrome 
204 (ACS). Subgroup analysis showed that the higher the FGF21 level was, the sooner the ACS 205 occurred and the higher the incidence of PCI. These studies suggested that for patients with 206 cardiovascular disease, elevated FGF21 levels indicated a poor prognosis. Similarly, serum 207 FGF21 levels were an independent predictor of incident coronary heart disease (CHD) in 208 diabetic patients (Lee C H, et al. 2017). In general, for patients with cardiovascular disease or 209 diabetes, FGF21 is likely to be a powerful biomarker for predicting MACEs. However, for 210 healthy people, a large number of studies are still needed to validate this conclusion.

211 Although several studies have focused on the association of FGF21 with AMI, we found for 212 the first time that FGF21 was related to the prognosis of STEMI patients after treatment with 213 emergency PCI. Sunaga et al found that serum FGF21 levels were significantly higher in patients 214 with AMI after PCI than in patients with SA, but this study was not followed up (Sunaga H, et al. 215 2019). Another study that included a sample of 55 STEMI and NSTEMI patients found that 216 FGF21 was associated with AMI and with the incidence of reinfarction within 30 days after 217 onset (Zhang W, et al. 2015). Similar to our study, Chen et al. found that FGF21 was associated 218 with MACEs in patients with AMI (Chen H, et al. 2018). However, unlike our study, the study 219 did not distinguish between STEMI patients and NSTEMI patients, although STEMI patients 220 were enrolled in the study. Overall, FGF21 was first identified as a MACE-related biomarker in 221 our follow-up study of STEMI patients treated with emergency PCI.

222 The study had several limitations. Our study was conducted at a single hospital and 223 included only Chinese people. Although our sample size meets the needs of the study, our 224 follow-up time was too short, and the number of endpoints was not large enough. The absence of 225 height, weight, ECG and peak troponin I data made it impossible to assess other factors 226 associated with MACEs. Unfortunately, we did not follow up the SA group and therefore could 227 not compare it to the STEMI group. In addition, serum FGF21 levels were measured only at 228 admission instead of including a repeated measurement at the time of readmission. Therefore, 229 large-scale and long-term clinical studies are needed to confirm the role of FGF21 and its 230 relationship to STEMI.

231 Moreover, early echocardiography was used to assess the prognosis of STEMI patients who 232 underwent emergency PCI. Although early echocardiography is not the best method to predict 233 outcomes, some studies show that early echocardiography has a predictive value for the 234 prognosis of AMI (Awad E M L, et al. 2020; Cetin M, et al. 2021; Reindl M, et al. 2019).

235 However, a better approach would be to perform an echocardiography at the follow-up visit with 236 a before-and-after comparison. 


\section{Conclusions}

239 In conclusion, we observed that serum FGF21 levels were significantly elevated in STEMI

240 patients who had undergone emergency PCI. Moreover, elevated FGF21 levels on admission

241 have been shown to be a powerful predictor of MACEs. Therefore, FGF21 can be used as a

242 novel biomarker for STEMI after emergency PCI.

243

\section{Acknowledgements}

245 The author thanks Professor Yao Yuyu for his assistance in this manuscript.

246

\section{References}

2481 Awad, EML, Mahmoud, AH, Maghrby, KS, Taha, NM,Ibrahim, AM. 2020. Short-term 249 prognostic value of TAPSE, RVFAC and Tricuspid S' wave peak systolic velocity after first

250

251

252

253

254

255

256

257

258

259

260

261

262

263

264

265

266

267

268

269

270

271

272

273

274

275 acute myocardial infarction. BMC Res Notes, 13: 196. DOI 10.1186/s13104-020-05040-2.

2 BonDurant, LD,Potthoff, MJ. 2018. Fibroblast Growth Factor 21: A Versatile Regulator of Metabolic Homeostasis. Annu Rev Nutr, 38: 173-96. DOI 10.1146/annurev-nutr-071816064800 .

3 Brahma, MK, Adam, RC, Pollak, NM, Jaeger, D, Zierler, KA, Pocher, N, Schreiber, R, Romauch, M, Moustafa, T, Eder, S, Ruelicke, T, Preiss-Landl, K, Lass, A, Zechner, R, Haemmerle, G. 2014. Fibroblast growth factor 21 is induced upon cardiac stress and alters cardiac lipid homeostasis. J Lipid Res, 55: 2229-41. DOI 10.1194/jlr.M044784.

4 Cetin, M, Ozer, S, Cinier, G, Yilmaz, AS, Erdogan, T,Satiroglu, O. 2021. Left atrial volume index and pulmonary arterial pressure predicted MACE among patients with STEMI during 8-year follow-up: experience from a tertiary center. Herz, 46: 367-74. DOI 10.1007/s00059020-04966-4.

5 Chen, H, Lu, N,Zheng, M. 2018. A high circulating FGF21 level as a prognostic marker in patients with acute myocardial infarction. Am J Transl Res, 10: 2958-66. DOI.

6 Domouzoglou, EM, Naka, KK, Vlahos, AP, Papafaklis, MI, Michalis, LK, Tsatsoulis, A, Maratos-Flier, E. 2015. Fibroblast growth factors in cardiovascular disease: The emerging role of FGF21. Am $J$ Physiol Heart Circ Physiol, 309: H1029-38. DOI 10.1152/ajpheart.00527.2015.

7 Fisher, FM,Maratos-Flier, E. 2016. Understanding the Physiology of FGF21. Annu Rev Physiol, 78: 223-41. DOI 10.1146/annurev-physiol-021115-105339.

8 Groot, HE, Hartman, MH, Gu, YL, de Smet, BJ, van den Heuvel, AF, Lipsic, E, van der Harst, P. 2015. Soluble interleukin 6 receptor levels are associated with reduced myocardial reperfusion after percutaneous coronary intervention for acute myocardial infarction. Cytokine, 73: 207-12. DOI 10.1016/j.cyto.2015.02.004.

9 Gu, L, Jiang, W, Zheng, R, Yao, Y,Ma, G. 2020. Fibroblast Growth Factor 21 Correlates with the Prognosis of Dilated Cardiomyopathy. Cardiology, 1-7. DOI 10.1159/000509239. 
27610 Gu, L, Jiang, W, Zheng, R, Yao, Y,Ma, G. 2021. Fibroblast Growth Factor 21 Correlates with 277 the Prognosis of Dilated Cardiomyopathy. Cardiology, 146: 27-33. DOI 10.1159/000509239.

$27811 \mathrm{Hu}, \mathrm{S}$, Cao, S, Tong, Z,Liu, J. 2018. FGF21 protects myocardial ischemia-reperfusion injury 279 through reduction of miR-145-mediated autophagy. Am J Transl Res, 10: 3677-88. DOI.

28012 Kuch, B, von Scheidt, W, Kling, B, Heier, M, Hoermann, A,Meisinger, C. 2007. 281 Characteristics and outcome of patients with acute myocardial infarction according to 282

283

284

285

286

287

288 presenting electrocardiogram (from the MONICA/KORA Augsburg Myocardial Infarction-Registry). Am J Cardiol, 100: 1056-60. DOI 10.1016/j.amjcard.2007.04.054.

13 Lakhani, I, Gong, M, Wong, WT, Bazoukis, G, Lampropoulos, K, Wong, SH, Wu, WKK, Wong, MCS, Ong, KL, Liu, T, Tse, G, International Health Informatics Study, N. 2018. Fibroblast growth factor 21 in cardio-metabolic disorders: a systematic review and metaanalysis. Metabolism, 83: 11-17. DOI 10.1016/j.metabol.2018.01.017.

14 Lang, RM, Badano, LP, Mor-Avi, V, Afilalo, J, Armstrong, A, Ernande, L, Flachskampf, FA,

289 Foster, E, Goldstein, SA, Kuznetsova, T, Lancellotti, P, Muraru, D, Picard, MH, Rietzschel,

290

291

292

293

294

295 ER, Rudski, L, Spencer, KT, Tsang, W, Voigt, JU. 2015. Recommendations for cardiac chamber quantification by echocardiography in adults: an update from the American Society of Echocardiography and the European Association of Cardiovascular Imaging. Eur Heart J Cardiovasc Imaging, 16: 233-70. DOI 10.1093/ehjci/jev014.

15 Lee, CH, Woo, YC, Chow, WS, Cheung, CYY, Fong, CHY, Yuen, MMA, Xu, A, Tse, HF,

296

297

298

299

300 Lam, KSL. 2017. Role of Circulating Fibroblast Growth Factor 21 Measurement in Primary Prevention of Coronary Heart Disease Among Chinese Patients With Type 2 Diabetes Mellitus. J Am Heart Assoc, 6. DOI 10.1161/JAHA.116.005344.

16 Lewis, JE, Ebling, FJP, Samms, RJ,Tsintzas, K. 2019. Going Back to the Biology of FGF21: New Insights. Trends Endocrinol Metab, 30: 491-504. DOI 10.1016/j.tem.2019.05.007.

301

17 Luo, Y, Ye, S, Chen, X, Gong, F, Lu, W,Li, X. 2017. Rush to the fire: FGF21 extinguishes metabolic stress, metaflammation and tissue damage. Cytokine Growth Factor Rev, 38: 59-

302

303 65. DOI 10.1016/j.cytogfr.2017.08.001.

304

305

306

18 Neri, M, Riezzo, I, Pascale, N, Pomara, C,Turillazzi, E. 2017. Ischemia/Reperfusion Injury following Acute Myocardial Infarction: A Critical Issue for Clinicians and Forensic

307 Pathologists. Mediators Inflamm, 2017: 7018393. DOI 10.1155/2017/7018393.

19 O'Gara, PT, Kushner, FG, Ascheim, DD, Casey, DE, Jr., Chung, MK, de Lemos, JA, Ettinger, SM, Fang, JC, Fesmire, FM, Franklin, BA, Granger, CB, Krumholz, HM, Linderbaum, JA,

309 Morrow, DA, Newby, LK, Ornato, JP, Ou, N, Radford, MJ, Tamis-Holland, JE, Tommaso,

310

311

312

313 CL, Tracy, CM, Woo, YJ, Zhao, DX. 2013. 2013 ACCF/AHA guideline for the management of ST-elevation myocardial infarction: a report of the American College of Cardiology Foundation/American Heart Association Task Force on Practice Guidelines. Journal of the American College of Cardiology, 61: e78-e140. DOI 10.1016/j.jacc.2012.11.019.

314

20 Ong, KL, Campbell, S, Wu, BJ, McClelland, RL, Kokkinos, J, Szklo, M, Polak, JF, Allison, MA, Rye, KA. 2019. Relationship of fibroblast growth factor 21 with subclinical 
315 atherosclerosis and cardiovascular events: Multi-Ethnic Study of Atherosclerosis. $316 \quad$ Atherosclerosis, 287: 46-53. DOI 10.1016/j.atherosclerosis.2019.06.898.

31721 Ong, SB, Hernandez-Resendiz, S, Crespo-Avilan, GE, Mukhametshina, RT, Kwek, XY, 318 Cabrera-Fuentes, HA, Hausenloy, DJ. 2018. Inflammation following acute myocardial 319 infarction: Multiple players, dynamic roles, and novel therapeutic opportunities. Pharmacol Ther, 186: 73-87. DOI 10.1016/j.pharmthera.2018.01.001.

321

22 Planavila, A, Redondo, I, Hondares, E, Vinciguerra, M, Munts, C, Iglesias, R, Gabrielli, LA, Sitges, M, Giralt, M, van Bilsen, M, Villarroya, F. 2013. Fibroblast growth factor 21 protects against cardiac hypertrophy in mice. Nature Communications, 4: 2019. DOI 10.1038/ncomms3019.

23 Planavila, A, Redondo-Angulo, I, Ribas, F, Garrabou, G, Casademont, J, Giralt, M, Villarroya, F. 2015. Fibroblast growth factor 21 protects the heart from oxidative stress. Cardiovascular Research, 106: 19-31. DOI 10.1093/cvr/cvu263.

327

24 Planavila, A, Redondo-Angulo, I,Villarroya, F. 2015. FGF21 and Cardiac Physiopathology.

329 Front Endocrinol (Lausanne), 6: 133. DOI 10.3389/fendo.2015.00133.

330

25 Reindl, M, Reinstadler, SJ, Tiller, C, Feistritzer, HJ, Kofler, M, Brix, A, Mayr, A, Klug, G,

331

332 Metzler, B. 2019. Prognosis-based definition of left ventricular remodeling after ST-elevation myocardial infarction. Eur Radiol, 29: 2330-39. DOI 10.1007/s00330-018-5875-3.

333

26 Shen, Y, Zhang, X, Pan, X, Xu, Y, Xiong, Q, Lu, Z, Ma, X, Bao, Y, Jia, W. 2017.

334

335

336

337 Contribution of serum FGF21 level to the identification of left ventricular systolic dysfunction and cardiac death. Cardiovasc Diabetol, 16: 106. DOI 10.1186/s12933-0170588-5.

27 Shen, Y, Zhang, X, Xu, Y, Xiong, Q, Lu, Z, Ma, X, Bao, Y, Jia, W. 2018. Serum FGF21 Is Associated with Future Cardiovascular Events in Patients with Coronary Artery Disease. Cardiology, 139: 212-18. DOI 10.1159/000486127.

340

28 Steven, S, Frenis, K, Oelze, M, Kalinovic, S, Kuntic, M, Bayo Jimenez, MT, Vujacic-Mirski,

341 K, Helmstadter, J, Kroller-Schon, S, Munzel, T, Daiber, A. 2019. Vascular Inflammation and Oxidative Stress: Major Triggers for Cardiovascular Disease. Oxid Med Cell Longev, 2019: 7092151. DOI 10.1155/2019/7092151.

345

29 Sunaga, H, Koitabashi, N, Iso, T, Matsui, H, Obokata, M, Kawakami, R, Murakami, M, Yokoyama, T, Kurabayashi, M. 2019. Activation of cardiac AMPK-FGF21 feed-forward loop in acute myocardial infarction: Role of adrenergic overdrive and lipolysis byproducts. Sci Rep, 9: 11841. DOI 10.1038/s41598-019-48356-1.

349

30 Tao, L, Bei, Y, Lin, S, Zhang, H, Zhou, Y, Jiang, J, Chen, P, Shen, S, Xiao, J, Li, X. 2015. Exercise Training Protects Against Acute Myocardial Infarction via Improving Myocardial Energy Metabolism and Mitochondrial Biogenesis. Cell Physiol Biochem, 37: 162-75. DOI 10.1159/000430342.

353

31 Wang, R, Yi, X, Li, X,Jiang, X. 2015. Fibroblast growth factor-21 is positively associated with atrial fibrosis in atrial fibrillation patients with rheumatic heart disease. Int J Clin Exp 
354

355

356

357

358

359

360

361

362

363

364

365

366

367

368

369

370

371

372

373

374

375

376

377

378

379

380

381

382

383

384

385

Pathol, 8: 14901-8. DOI.

32 Woo, YC, Xu, A, Wang, Y,Lam, KS. 2013. Fibroblast growth factor 21 as an emerging metabolic regulator: clinical perspectives. Clin Endocrinol (Oxf), 78: 489-96. DOI 10.1111/cen.12095.

$33 \mathrm{Wu}, \mathrm{CH}$, Chou, RH, Kuo, CS, Huang, PH, Chang, CC, Leu, HB, Huang, CC, Chen, JW, Lin, SJ. 2018. Circulating Fibroblast Growth Factor 21 is Associated with Subsequent Renal Injury Events in Patients Undergoing Coronary Angiography. Sci Rep, 8: 12425. DOI 10.1038/s41598-018-30744-8.

34 Zhang, J, Cheng, Y, Gu, J, Wang, S, Zhou, S, Wang, Y, Tan, Y, Feng, W, Fu, Y, Mellen, N, Cheng, R, Ma, J, Zhang, C, Li, Z, Cai, L. 2016. Fenofibrate increases cardiac autophagy via FGF21/SIRT1 and prevents fibrosis and inflammation in the hearts of Type 1 diabetic mice. Clin Sci (Lond), 130: 625-41. DOI 10.1042/CS20150623.

35 Zhang, Q, Shao, M, Zhang, X, Wang, Q, Guo, D, Yang, X, Li, C, Wang, Y. 2018. The Effect of Chinese Medicine on Lipid and Glucose Metabolism in Acute Myocardial Infarction Through PPARgamma Pathway. Front Pharmacol, 9: 1209. DOI 10.3389/fphar.2018.01209.

36 Zhang, W, Chu, S, Ding, W,Wang, F. 2015. Serum Level of Fibroblast Growth Factor 21 Is Independently Associated with Acute Myocardial Infarction. PLoS One, 10: e0129791. DOI 10.1371/journal.pone.0129791.

37 Zhu, W, Wang, C, Liu, L, Li, Y, Li, X, Cai, J, Wang, H. 2014. Effects of fibroblast growth factor 21 on cell damage in vitro and atherosclerosis in vivo. Can J Physiol Pharmacol, 92: 927-35. DOI 10.1139/cjpp-2014-0227.

38 Ziakas, A, Gavrilidis, S, Giannoglou, G, Souliou, E, Gemitzis, K, Kalampalika, D, Vayona, MA, Pidonia, I, Parharidis, G, Louridas, G. 2006. In-hospital and long-term prognostic value of fibrinogen, CRP, and IL-6 levels in patients with acute myocardial infarction treated with thrombolysis. Angiology, 57: 283-93. DOI 10.1177/000331970605700304.

39 Zoghbi, WA, Enriquez-Sarano, M, Foster, E, Grayburn, PA, Kraft, CD, Levine, RA, Nihoyannopoulos, P, Otto, CM, Quinones, MA, Rakowski, H, Stewart, WJ, Waggoner, A, Weissman, NJ, American Society of, E. 2003. Recommendations for evaluation of the severity of native valvular regurgitation with two-dimensional and Doppler echocardiography. $J$ Am Soc Echocardiogr, 16: 777-802. DOI 10.1016/S08947317(03)00335-3. 


\section{Figure 1}

Flow chart of this study

A total of 348 patients with STEMI were enrolled from January 2016 to December 2018. They all underwent emergency coronary angiography and $\mathrm{PCl}$ within 12 hours after the onset of ischemic symptoms. 80 patients with stable angina who underwent selective coronary angiography and $\mathrm{PCl}$ were selected as the control group. Follow-up, which was initiated at enrollment and ended in April 2020, was conducted for STEMI patients only. The median follow-up time was 24 months (interquartile range, 16-36.25 months). The primary endpoint of the study was the first MACE, defined as all-cause mortality or readmission for angina, heart failure, or recurrent AMI. Eventually, 30 of the STEMI patients were lost to follow-up and 106 recorded MACEs, including 6 deaths and 100 readmissions. $\mathrm{PCl}$ : percutaneous coronary intervention; STEMI: st-segment elevation myocardial infarction; MACEs: major adverse cardiovascular events; AMI: acute myocardial infarction. 
Participation after emergency or selective $\mathrm{PCl}$ (between January 2016 and December 2018)

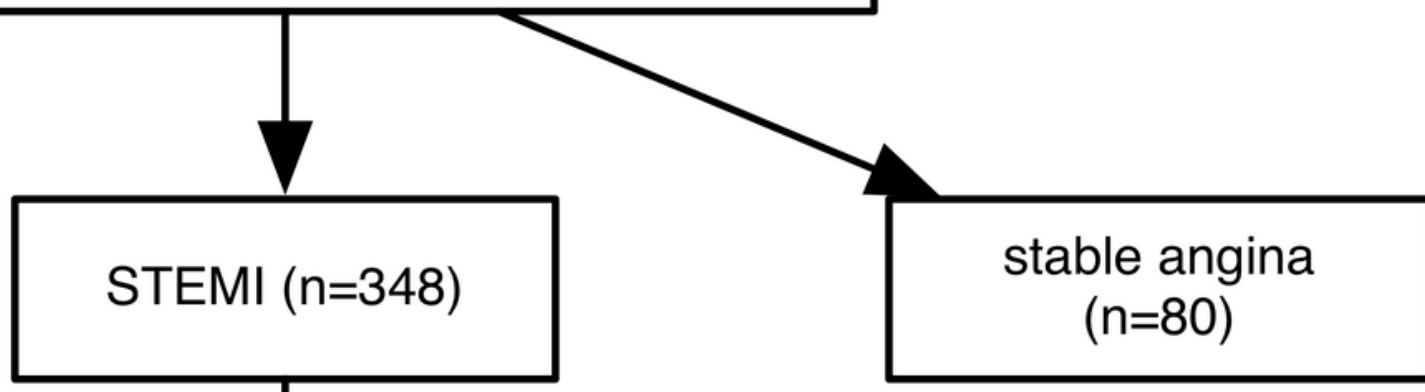

During the median follow-up of 24 months, 106 patients experienced MACEs

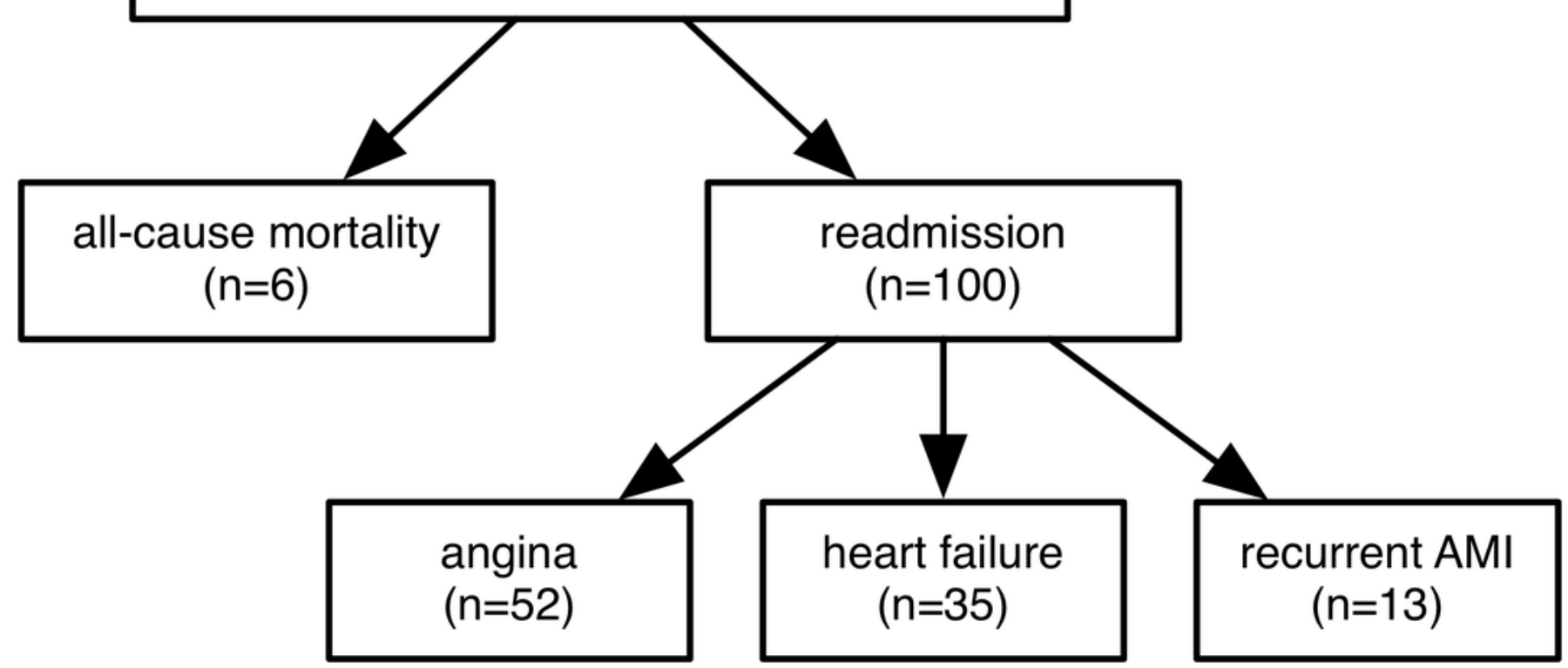




\section{Figure 2}

Receiver operating characteristic curve for predicting prognosis in STEMI patients after emergency $\mathrm{PCl}$

During the median follow-up of 24 months, 106 patients experienced MACEs in the study: 6 patients died, and 100 patients were rehospitalized due to angina, heart failure or recurrent AMI. According to the ROC analysis, both FGF21 and NT-proBNP levels were significant indicators of MACEs in STEMI patients. The area under the ROC curve (AUC) of NT-proBNP was 0.865 (95\% Cl: $0.822-0.909$ ), which was greater than that of FGF21 (AUC, $0.812 ; 95 \% \mathrm{Cl}$ : 0.765-0.860). The difference between the two AUCs was statistically significant $(Z=2.230$, $\mathrm{P}=0.026$ ). NT-proBNP, N-terminal proB-type natriuretic peptide; FGF21, fibroblast growth factor 21 . 


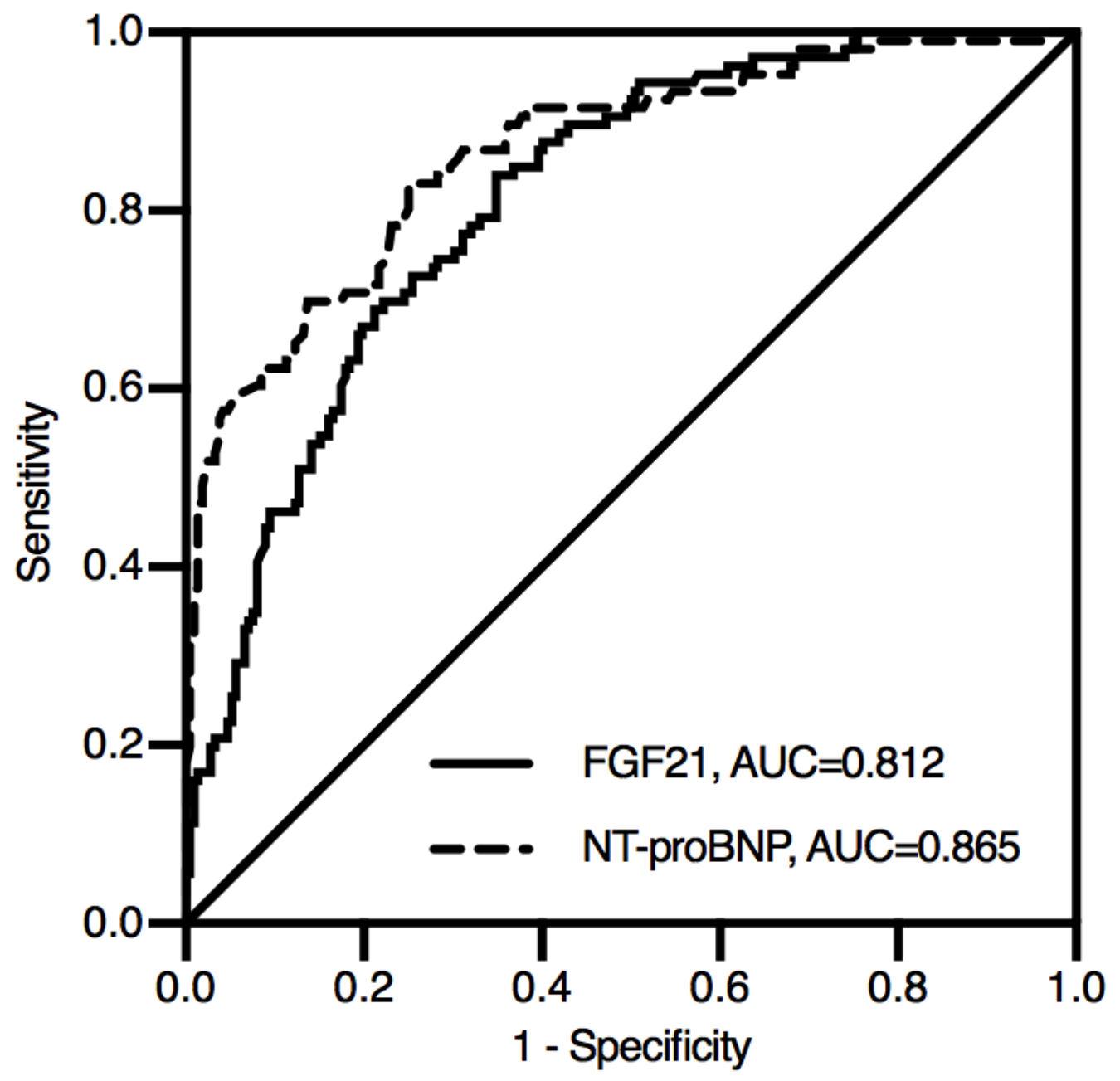


Figure 3

Kaplan-Meier curve showing the Mace-free probability of STEMI patients after emergency PCl with different FGF21 levels.

According to the optimal cutoff value determined by ROC analysis and Youden index, FGF21 was divided into high FGF21 level group and low FGF21 level group. The optimal cutoff value of FGF21 was $229.77 \mathrm{pg} / \mathrm{ml}$. The Mace-free probability among STEMI patients with high serum FGF21 levels was significantly lower than that of STEMI patients with low serum FGF21 levels.

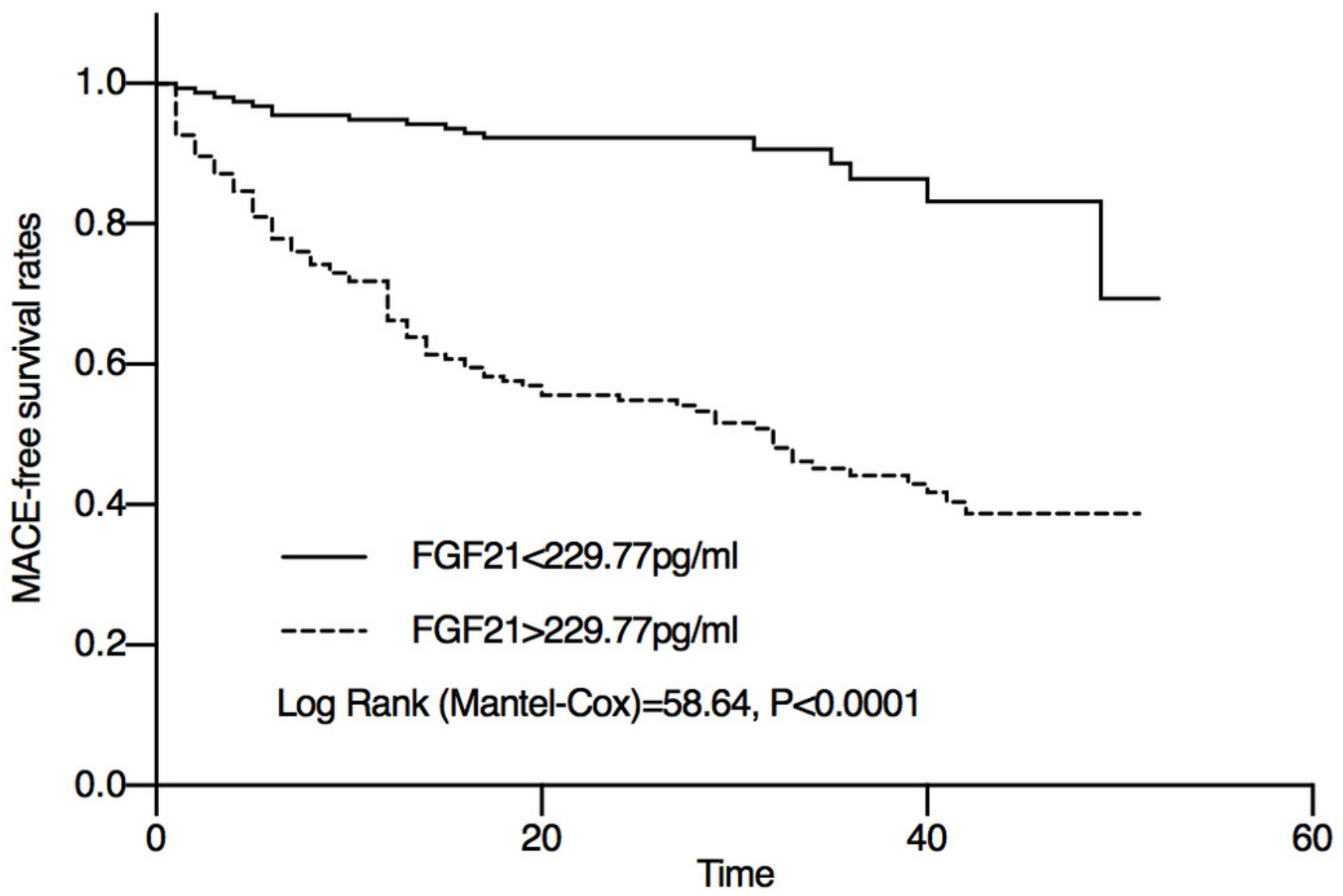




\section{Table $\mathbf{1}$ (on next page)}

The Demographic and Baseline Clinical Characteristics of the Patients

LDL-C, low density lipoprotein cholesterol; HDL-C, high density lipoprotein cholesterol; NTproBNP, N-terminal proB-type natriuretic peptide; FGF21, fibroblast growth factor 21; IVST, interventricular septal wall thickness; LVPWT, left ventricular posterior wall thickness; LVEDD, left ventricular end-diastolic diameter; LVESD, left ventricular systolic diameter; LVEDV, left ventricular end-diastolic volume; LVESV, left ventricular end-systolic volume; LVM, left ventricular mass; LVEF, left ventricular ejection fraction; LAD, left atrial dimension; LCX, left circumflex artery; RCA, right coronary artery. 
1 Table 1:

2 The Demographic and Baseline Clinical Characteristics of the Patients

\begin{tabular}{|c|c|c|c|}
\hline Variables & $\begin{array}{l}\text { STEMI group } \\
(\mathrm{n}=348)\end{array}$ & $\begin{array}{l}\text { SA group } \\
(n=80)\end{array}$ & $P$ value \\
\hline \multicolumn{4}{|l|}{ Demographic data } \\
\hline Male, n (\%) & $280(80.46 \%)$ & $57(71.25 \%)$ & 0.069 \\
\hline Age (years) & $62.05 \pm 13.05$ & $59.70 \pm 12.56$ & 0.144 \\
\hline Hypertension, n (\%) & $185(53.16 \%)$ & $17(21.25 \%)$ & 0.020 \\
\hline Diabetes mellitus, n (\%) & $64(18.39 \%)$ & $7(8.75 \%)$ & 0.037 \\
\hline Atrial fibrillation, n (\%) & $27(7.76 \%)$ & $4(5.00 \%)$ & 0.391 \\
\hline \multicolumn{4}{|l|}{ Laboratory data } \\
\hline Total cholesterol (mmol/L) & $4.33 \pm 1.11$ & $4.50 \pm 1.29$ & 0.271 \\
\hline Triglyceride (mmol/L) & $1.83 \pm 1.44$ & $2.04 \pm 1.57$ & 0.245 \\
\hline $\mathrm{LDL}-\mathrm{C}(\mathrm{mmol} / \mathrm{L})$ & $3.26 \pm 1.01$ & $2.49 \pm 0.72$ & 0.000 \\
\hline HDL-C (mmol/L) & $1.14 \pm 0.44$ & $1.29 \pm 0.42$ & 0.008 \\
\hline Uric acid $(\mu \mathrm{mol} / \mathrm{L})$ & $340.16 \pm 98.12$ & $332.52 \pm 59.90$ & 0.370 \\
\hline Creatinin $(\mu \mathrm{mol} / \mathrm{L})$ & $80.88 \pm 59.96$ & $72.18 \pm 20.05$ & 0.219 \\
\hline Troponin I (ng/ml) & $44.07 \pm 20.76$ & - & - \\
\hline NT-proBNP (pg/ml) & $593.17 \pm 584.25$ & $164.03 \pm 100.25$ & 0.000 \\
\hline FGF21 (pg/ml) & $225.03 \pm 37.98$ & $135.51 \pm 34.48$ & 0.000 \\
\hline \multicolumn{4}{|l|}{ Echocardiographic data } \\
\hline Pulmonary pressure (mmHg) & $32.30 \pm 8.34$ & $32.00 \pm 6.16$ & 0.762 \\
\hline Mitral regurgitation & $49(14.08 \%)$ & $11(13.75 \%)$ & 0.818 \\
\hline LAD (mm) & $41.26 \pm 4.74$ & $39.68 \pm 4.05$ & 0.006 \\
\hline IVST (mm) & $9.75 \pm 1.69$ & $9.63 \pm 1.33$ & 0.548 \\
\hline LVPWT (mm) & $9.86 \pm 1.35$ & $9.65 \pm 1.25$ & 0.203 \\
\hline LVEDD (mm) & $52.42 \pm 4.45$ & $50.33 \pm 3.96$ & 0.000 \\
\hline LVESD (mm) & $37.75 \pm 4.83$ & $33.18 \pm 4.07$ & 0.000 \\
\hline LVEDV (ml) & $127.18 \pm 38.03$ & $121.08 \pm 21.89$ & 0.057 \\
\hline LVESV (ml) & $59.63 \pm 23.52$ & $45.76 \pm 14.58$ & 0.000 \\
\hline $\operatorname{LVM}(\mathrm{g})$ & $193.21 \pm 42.47$ & $177.35 \pm 40.14$ & 0.003 \\
\hline LVEF (\%) & $53.66 \pm 7.99$ & $62.36 \pm 6.32$ & 0.000 \\
\hline \multicolumn{4}{|l|}{ Angiographic data } \\
\hline Culprit artery & & & 0.003 \\
\hline LAD, n (\%) & $207(59.48 \%)$ & $61(76.25 \%)$ & \\
\hline LCX, n (\%) & $32(9.20 \%)$ & $7(8.75)$ & \\
\hline
\end{tabular}




\begin{tabular}{clll}
\hline RCA, n (\%) & $109(31.32 \%)$ & $12(15.00 \%)$ & \\
Culprit lesion site & & & 0.038 \\
Proximal segment, n (\%) & $195(56.03 \%)$ & $32(40.00 \%)$ & \\
Middle segment, n (\%) & $121(34.77 \%)$ & $43(53.75 \%)$ & \\
Distal segment, n (\%) & $32(9.20 \%)$ & $5(6.25)$ & \\
Number of diseased vessels & & & 0.194 \\
One vessels, n (\%) & $248(71.26 \%)$ & $62(77.50 \%)$ & \\
Two vessels, n (\%) & $67(19.25 \%)$ & $15(18.75 \%)$ & \\
Three vessels, n (\%) & $33(9.48 \%)$ & $3(3.75 \%)$ & \\
\hline
\end{tabular}

3 LDL-C, low density lipoprotein cholesterol; HDL-C, high density lipoprotein cholesterol; NT-proBNP, N-

4 terminal proB-type natriuretic peptide; FGF21, fibroblast growth factor 21; IVST, interventricular septal wall

5 thickness; LVPWT, left ventricular posterior wall thickness; LVEDD, left ventricular end-diastolic diameter;

6 LVESD, left ventricular systolic diameter; LVEDV, left ventricular end-diastolic volume; LVESV, left

7 ventricular end-systolic volume; LVM, left ventricular mass; LVEF, left ventricular ejection fraction; LAD,

8 left atrial dimension; LCX, left circumflex artery; RCA, right coronary artery. 


\section{Table 2 (on next page)}

Univariate and Multivariate COX Analysis for Prognosis in STEMI Patients

LDL-C, low density lipoprotein cholesterol; HDL-C, high density lipoprotein cholesterol; NTproBNP, N-terminal proB-type natriuretic peptide; FGF21, fibroblast growth factor 21; IVST, interventricular septal wall thickness; LVPWT, left ventricular posterior wall thickness; LVEDD, left ventricular end-diastolic diameter; LVESD, left ventricular systolic diameter; LVEDV, left ventricular end-diastolic volume; LVESV, left ventricular end-systolic volume; LVM, left ventricular mass; LVEF, left ventricular ejection fraction; LAD, left atrial dimension; LCX, left circumflex artery; RCA, right coronary artery. 
1 Table 2:

2 Univariate and Multivariate COX Analysis for Prognosis in STEMI Patients

\begin{tabular}{|c|c|c|c|c|c|c|}
\hline \multirow[t]{2}{*}{ Variables } & \multicolumn{3}{|c|}{ Univariate analysis } & \multicolumn{3}{|c|}{ Multivariate analysis } \\
\hline & HR & $95 \% \mathrm{CI}$ & $P$ value & HR & $95 \% \mathrm{CI}$ & $P$ value \\
\hline Sex (male) & 0.744 & $0.474-1.166$ & 0.197 & - & - & - \\
\hline Age (years) & 1.766 & $1.188-2.626$ & 0.005 & - & - & - \\
\hline Hypertension & 1.102 & $0.752-1.616$ & 0.619 & - & - & - \\
\hline Diabetes mellitus & 1.398 & $0.874-2.236$ & 0.162 & - & - & - \\
\hline Atrial fibrillation & 1.317 & $0.705-2.461$ & 0.388 & & & \\
\hline Total cholesterol & 0.872 & $0.728-1.045$ & 0.139 & - & - & - \\
\hline Triglyceride & 0.850 & $0.718-1.006$ & 0.059 & - & - & - \\
\hline LDL-C & 1.168 & $0.965-1.414$ & 0.111 & - & - & - \\
\hline HDL-C & 0.773 & $0.479-1.245$ & 0.289 & - & - & - \\
\hline Uric acid & 1.000 & $0.998-1.001$ & 0.676 & - & - & - \\
\hline Creatinine & 1.002 & $1.000-1.004$ & 0.016 & - & - & - \\
\hline Troponin I & 8.143 & $4.834-14.716$ & 0.000 & 6.842 & $3.870-12.094$ & 0.000 \\
\hline NT-proBNP & 7.496 & $4.508-12.462$ & 0.000 & 3.452 & $1.765-6.750$ & 0.000 \\
\hline FGF21 & 5.901 & $3.509-9.922$ & 0.000 & 2.224 & $1.122-4.407$ & 0.022 \\
\hline Pulmonary pressure & 1.510 & $1.015-2.247$ & 0.042 & & & \\
\hline Mitral regurgitation & 1.745 & $1.071-2.844$ & 0.025 & 2.011 & $1.160-3.489$ & 0.013 \\
\hline LAD & 1.732 & $1.178-2.547$ & 0.005 & & & \\
\hline IVST & 1.381 & $0.671-2.842$ & 0.381 & & & \\
\hline LVPWT & 0.987 & $0.662-1.471$ & 0.948 & & & \\
\hline LVEDD & 1.359 & $0.924-1.998$ & 0.119 & & & \\
\hline LVESD & 1.749 & $1.170-2.614$ & 0.006 & & & \\
\hline LVEDV & 1.359 & $0.924-1.998$ & 0.119 & & & \\
\hline LVESV & 1.749 & $1.170-2.614$ & 0.006 & & & \\
\hline LVM & 1.334 & $0.882-2.016$ & 0.172 & & & \\
\hline LVEF & 1.999 & $1.339-2.985$ & 0.001 & & & \\
\hline \multicolumn{7}{|l|}{ Culprit artery } \\
\hline LAD & & & 0.077 & & & \\
\hline LCX & 0.487 & $0.212-1.120$ & 0.091 & & & \\
\hline $\mathrm{RCA}$ & 0.671 & $0.426-1.0574$ & 0.085 & & & \\
\hline \multicolumn{7}{|l|}{ Culprit lesion site } \\
\hline Proximal segment & 4.095 & $1.287-13.028$ & 0.017 & & & \\
\hline Middle segment & 3.660 & $1.127-11.892$ & 0.031 & & & \\
\hline
\end{tabular}


Distal segment

Number of diseased

vessels

One vessels

0.186

Two vessels

$$
1.144 \quad 0.921-2.262 \quad 0.109
$$

Three vessels

$0.794 \quad 0.383-1.649$

0.794

3 LDL-C, low density lipoprotein cholesterol; HDL-C, high density lipoprotein cholesterol; NT-proBNP, N-

4 terminal proB-type natriuretic peptide; FGF21, fibroblast growth factor 21; IVST, interventricular septal wall

5 thickness; LVPWT, left ventricular posterior wall thickness; LVEDD, left ventricular end-diastolic diameter;

6 LVESD, left ventricular systolic diameter; LVEDV, left ventricular end-diastolic volume; LVESV, left

7 ventricular end-systolic volume; LVM, left ventricular mass; LVEF, left ventricular ejection fraction; LAD,

8 left atrial dimension; LCX, left circumflex artery; RCA, right coronary artery. 